\title{
Review of Therapeutic Applications of Nanotechnology in Medicine Field and its Side Effects
}

\author{
Wanisa Abdussalam-Mohammed ${ }^{\mathrm{a}, *}$ \\ ${ }^{a}$ Department of Chemistry, College of Science, Sebha University, Sebha, Libya
}

\begin{abstract}
Receive Date: 02 June 2019, Revise Date: 23 July 2019, Accept Date: 30 July 2019
Abstract:

Nanotechnology is the new field of technological innovation on their application in human biology and medicine. On the other hand, there is an important concern about the potential health and environmental risks, due to exposure to human body of nanoparticles. These nanoparticles led to the appearance in the scientific disciplines such as nanotoxicology and nanomedicine. Nanotoxicology is the study toxicity effects of nanoparticles on human health as well as environment. Nano-particles have been developed for beneficial studies with applications in medical field and devices. Also, nanomedicines have been applied to the sector of molecular biology, tissue culture, bio-materials, genetic engineering, bio and bio-imaging sensors. Actually, nanotechnologies have followed healthcare improvements including potentially revolutionary treatments for cancer, critical diseases and also drug improvements with reduction of side effect. With all these descriptions, the extent of potential impact on human health is still a major concern due to lack of reliable toxicity data of nanoparticles. The main purpose of present literature is providing of better understanding on nanobiotechnology principles and its applications in biomedicine have been discussed as well as to their side effects.
\end{abstract}

DOI: $10.33945 /$ SAMI/JCR.2019.3.5

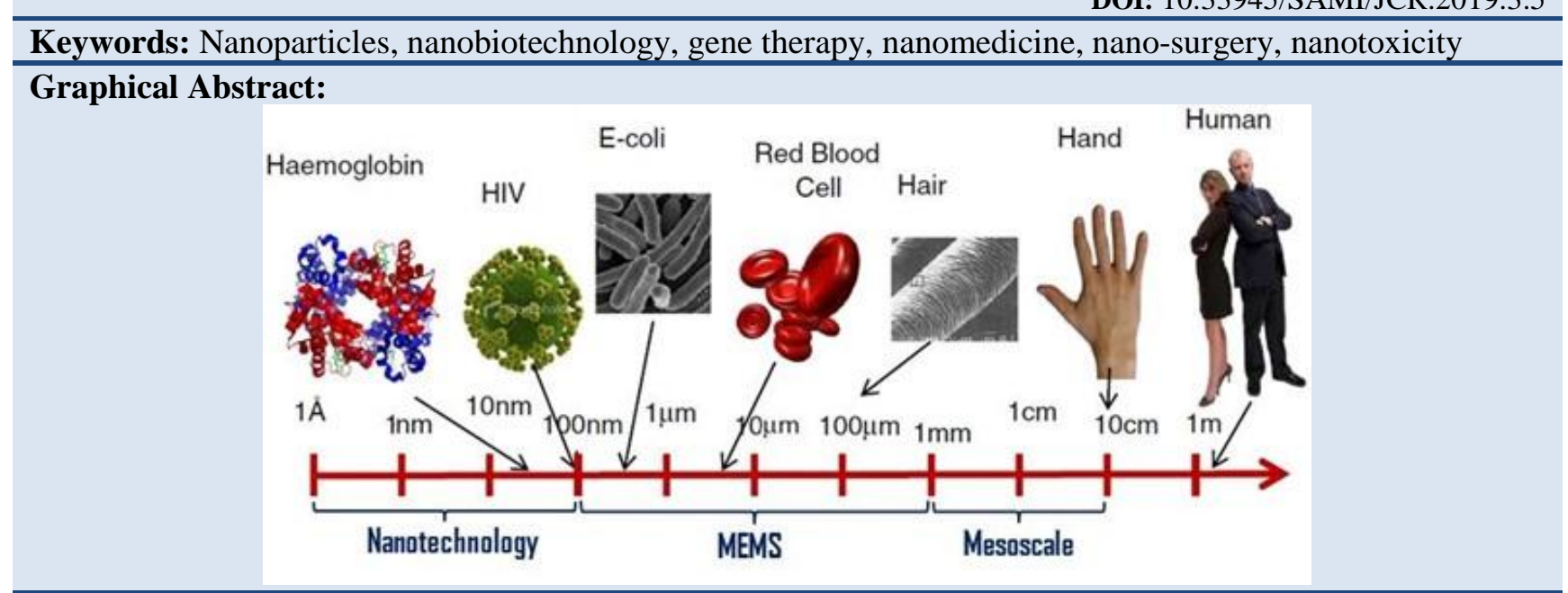

\section{Biography:}

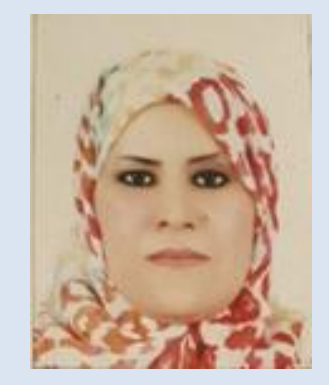

Wanisa Abdussalam-Mohammed was born in Marj (Libya). She has graduated from Sebha University (Libya) in 1998. She received her M.Sc. degree in 2005 in Organic Chemistry from Sebha University/ Libya. She earned her PhD in Bionanotechnology, from College of Engineering, Swansea University at United Kingdom 2017. Currently, she is working as a lecture at the Chemistry Department, Sebha University and cooperative with Chemical Engineering, Tripoli University, Libya. Her research interests are in the area of the synthesis of heterocyclic compounds and new synthetic methodologies. Prepetition of nanoparticles and using organic compounds to function metal nanoparticles. 


\section{Introduction}

\subsection{What is nanotechnology?}

Nano is known to be as a prefix meaning one-billionth. of a meter, or $10^{-9}$ meters and it is abbreviated "nm.". The nanometer is one-billionth of a meter which much too small in order to see with the naked eye or even with a traditional light microscope. For instance, a sheet of paper is about 100,000 nano-meters thick; while a single gold atom is about a third of a nanometer in diameter. Dimensions between 1 and 100 nano-meters are known as the nanoscale [1].

Nanotechnology has been employing materials at the nano scale, which considers a relatively new area for researchers, with rapidly increasing marketable applications which hold the promise of providing important improvements in technologies for protecting the environment and humanity. These technologies are being used in new and exciting applications in several different fields, such as medicine, textiles, environmental remediation and computer chip manufacturing [2-5].

Furthermore, it well to mention that nanoscience and nanotechnology not actually new things in the scientific world. For instance, in the past 50 years, a blooming of immunology caused to several new challenges. Continuous developments drove researchers to the years of molecular biology and genomics, which is a band of micro-science and microtechnology. Currently, nanotechnology focus on materials which have size from 1 to $100 \mathrm{~nm}$. Where at these sizes, materials have new advents which assistance researchers to control the accuracy and precision of new nano-materials usage [2,6-8]. Likewise, with nano size unique phenomena enable novel applications not feasible in case working with bulk materials with molecules or even single atoms.

\subsection{History and development of nanotechnology}

The term nanotechnology has been explained by a wide spectrum of numerous technologies that nanotechnology covers, which are based on many kinds of chemical, physical, and biological processes realised at Nano-level $[9,10]$. The accurately established time span for the beginning of nanotechnology development is demonstrated by the fact that nanotechnology has its backgrounds in the remote past when were used by people without knowledge of it.

The name "nanotechnology" was introduced by Norio Taniguchi for the first time in Tokyo in 1974 at the International Conference on Industrial Production in order to explain the super thin processing of materials with nanometer accuracy and the establishment of Nano-sized mechanisms. Concepts of nanotechnological approach, which were put forward by Richard Feynman (identified as Father of
Nanotechnology) in 1959 in his lecture delivered at the session of the American Physical Society and were developed in 1986 by Eric Drexler [9].

Nanotechnology and Nanoscience got an improvement in the early 1980s with two main developments including both the birth of cluster science and the invention of the Scanning Tunneling Microscope (STM) in 1981. These developments caused to the discovery of Fullerenes in 1985 and when Carbon Nano-tubes was advanced by Japanese scientist in 1991. In the medial of 1980s and beginning 1990s several significant discoveries were made, which had an essential impact on the additional development of nanotechnology. For example, in 1991, the first nanotechnological program of National Scientific Fund was started to operate in USA, and then in 2001, the National Nanotechnological Initiative (NNI) of the USA was approved. Since then, lots of technical research developments and scientific departments have taken place all over the world particularly in some countries such as England, Japan, China, Germany, France, South Korea and recently in the CIS countries [9-11].

Consequent, the nanotechnology pattern was formed at the turn of the 1960s, whereas the 1980s and 1990s are the start of growth of nanotechnology in its own right. According to the light of the toxicity testing in the 21st century suggested by the US National Research Council (NRC), high-throughput screening of nanomaterials looks promising and may be potential in the not too distant future. Despite of the complex nature of the nanomaterials which makes the development of their safety assessment challenging, the future of the nanotechnology sounds to be bright [12].

\subsection{Nanobiotechnology}

Nanobiotechnology is biotechnology at the nano scale and it is term that refer to the intersection of biology and nanotechnology, also it is a discipline in which tools from nanotechnology are developed and applied to study biological phenomena. For instance, nanoparticles can be serving as probes, sensors or vehicles for biomolecules delivery in cellular systems [11].

\subsubsection{Nanomedicine}

Nanomedicine is known as a new branch of medical science and holds five main sub-disciplines including: medical diagnosis, nanoimaging, chemotherapy analytical tools, nanodevices, and drug delivery systems. It can be said that the potential benefits of nanobiotechnologies raise great hopes in therapies for cancer diseases, antiviral and antifungal agents, diabetes, chronic lung diseases, and gene therapy. 
Besides medicine therapy, nanomedicine can be applied in surgery such as photodynamic therapy [11].

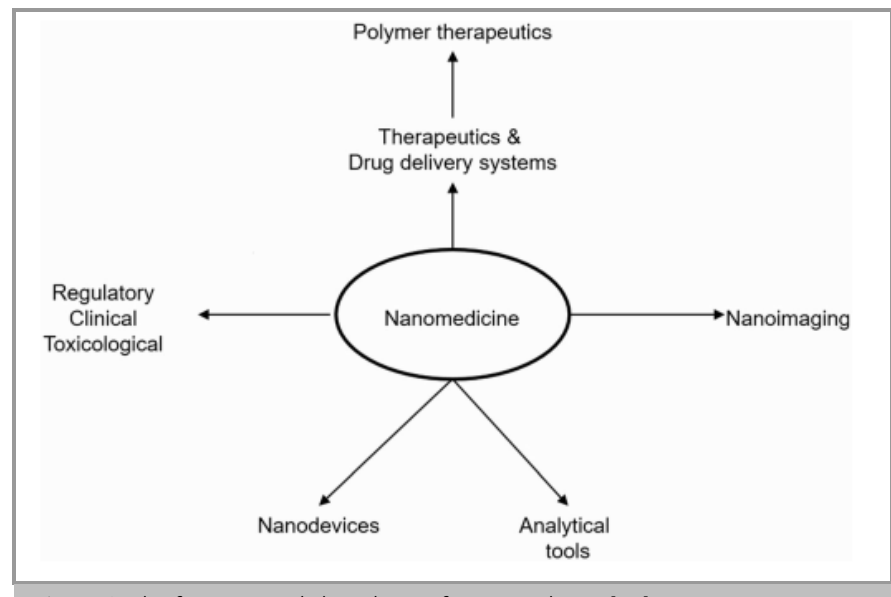

Figure 1. The five main subdisciplines of nanomedicine [11]

\subsubsection{Nanomedicine for Diagnosis}

Nanotechnologies can assistance physicians' diagnosis at single cell and molecule levels [12].

Nanoparticles, such as silver nanoparticles, gold nanoparticles, and quantum dots are the common used. While, various other nanotechnological devices for manipulation at the nanoscale as nanobio-sensors are also valuable for potential clinical applied usages [13]. In addition, nanotechnologies will provide the limits of present molecular diagnostics and assist in point of care diagnostics, combination of diagnostics with therapeutics, and development of personalised medicine $[11,13]$. Despite of the potential diagnostic applied usages are not limited, the most significant current applied usages are expected in the areas of biomarker discovery, early cancer diagnosis, and detection of infectious germs $[13,14]$.

\subsubsection{Nanomedicine for Therapy}

Treatment is a significant medical activity. Although it is preferred that prevention of diseases is better than treatment, but therapy for an already existing disorder is wanted [15-17]. Therapy can be also specific or symptomatic treatment; the main goal of treatment is to reduce of the patient's pain. Development of treatment has been continuous for centuries. The advent of nanotherapy in medical treatment field has begun to be a new hope in medicine [10,18-20].

The application of nanomaterials as a new supplement in order to the improvement of the conventional therapeutic protocol can be very beneficial $[10,21,22]$.

\subsubsection{Nano-pharmacology for nano-therapy}

With the advent on nano-pharmacology, new medicine development becomes easier in comparison with previous days, where the pharmaceutical process obtains a lot of worth from the new nano delivery system. Nano-pharmacology can be applied to nanotherapy and it is considered as the hope for treatment of presently untreatable diseases like cancer and human immunodeficiency virus $[23,24]$.

\subsubsection{Nano-surgery}

Nano-surgery is the new concept in surgical medicine [25-26]. As known that, a small wound is a wanted outcome of present surgery, where a small surgical wound means small blood loss and minimisation the complication of intraoperative and postoperative. A small wound also reduces the postoperative hospitalisation period for the patient. For example, there are some advents in eye surgery. Femtosecond laser pulses, which is emitted from lasers working in the near infrared, based on multiphoton effects allowing both imaging and laser effects to be produced which are in the sub-micron range and do not cause any collateral damage are available [27]. According to the literature, near-infrared femtosecond laser pulses was used for a collection of microscopy and nano-surgery on fluorescently labelled structures inside living cells [28]. It found that nano-surgery could be performed with submicrometer precision and without visible damage to the cell $[25,27,28]$.

In addition, nano neurosurgery is considered as another interesting emerging nano-surgery [29-31]. Also, several advents in nano-diagnosis can be applied to nano-surgery [25,29]. Targeted nano-therapy based on nano-pharmacology is also found to be valuable in nano-surgery [27, 30]. Furthermore, the applied usage of nanobiotechnology for nano-surgery can also be easily described as the development of various nanosurgical tools. Although bone is a very diverse tissue providing different functions within the body, recent researches have caused new biomaterials with promise to solve orthopedic problems $[25,32]$.

\subsubsection{Nanomaterials and Chemotherapy}

Using of unique nanomaterials in drug delivery has offered many advantages, such as increase of drug solubility, stability, and enhancement of drug half-lives in the blood circulation with little effects to healthy cells. Liposomes is an important nanomaterial as a phospholipid vesicle with a bilayered membrane structure and has been extensively used for delivering anticancer drugs. For example, PEGylated liposome can encapsulate doxorubicin for chemotherapy and be surface-functionalised by fluorophores or gadolinium for imaging [32].

Cancer is still the leading cause of death around the world, despite the many treatments developed over the past 30 years. Nanotechnology has contributed to important advances in cancer treatment via targeting, 
detecting, and destroying cancer cells with minimal effect on healthy tissues [33].

In addition, nanotechnology-based delivery system offers several advantages to conventional chemotherapy treatment, such as delivery of a higher dose of drugs to the tumour cells and improved solubility of unwell soluble drugs [11,34].

There are two important issues in developing nanomaterials used for cancer treatment: identification of the tumour and ability of the nanomaterials in order to reach the tumour site desired. Also, established and functionalised nanomaterial can convey novel advantages for cancer therapy, such as enhancing the efficiency of drugs delivered to wanted areas via declining the toxicity and side effects of the drugs. Functionalised nanomaterials should be having ability to carry a high concentration of imaging agents or chemotherapeutic agents as well as to targeting moieties to the site of tumours, which are connected with blood vessels $[11,35,36]$. Some of the chemical structure of some anticancer drugs, including Doxorubicin (DOX), Cisplatin, Cytarabine are showed in Scheme 1, these drugs can be conjugated to nanomaterials to increase their bioavailability and water solubility [37,38].

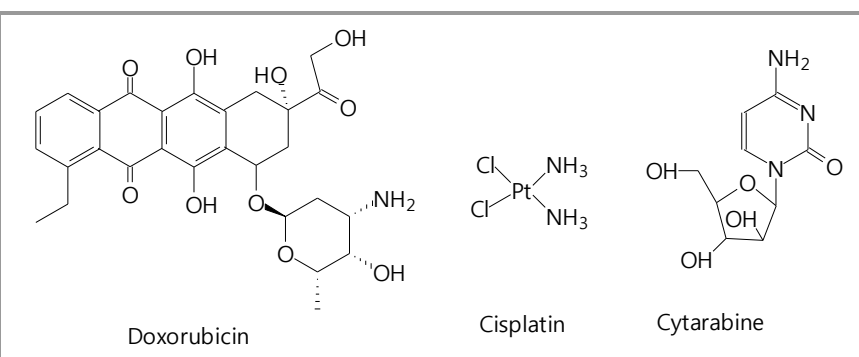

Scheme 1. Chemical structure of some anticancer drugs such as, Doxorubicin (DOX), Cisplatin, and Cytarabine [36]

It is well to mention that the synthesis of 2-pyridone nucleus derivatives is considered a challenge to the medicinal and synthetic chemists as its derivatives have significant biological and pharmacological activities [39]. For instance, a greener method has been used to synthesise 3,4-dihydropyridin-2-one derivatives which has some limitations and advantages such as shorter reaction times, simple work-up and excellent yield [39, 40]. These compounds were used as anticancer agents, spasmolytics, anti-coagulants, anti-anaphylactics [39].

In addition, due to anti-cancer properties of Curcumin (see Scheme 2), it has been used in traditional medicine for a varied range of ailments, including wound healing, liver ailments and urinary tract infection. To improve the medicinal value of curcumin in cancer prevention and treatment, novel nano formulations of curcumin have been synthesised, ranging from nanoparticles, nano-lipids, chitosan, and nanofibers. Lately, much attention has been directed to study the therapeutic application of Curcumin in the treatment of human cancer and the recent methods of curcumin preparation was used nanotechnology and have shown the remarkable therapeutic effects of prepared NPs of curcumin encased poly (lactic-co-glycolic acid) with a particle size of 35-100 nm and was more efficient on the prostate cancer cell line [41].

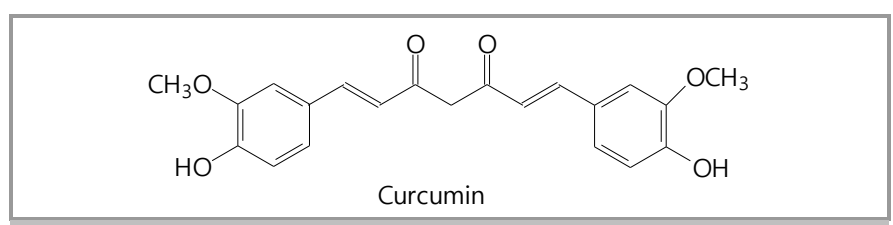

Scheme 2. Chemical structure of Curcumin [41]

\subsubsection{Nano-dentistry}

Nanotechnology has a significant participation in dentistry materials. For instance, (Ultra MicroIndentation System, UMIS-2000, CSIRO, Australia) was used as a nano-based indentation system to describe the indentation stress-strain response of two kinds of dental ceramics, one type of dental alloy and healthy enamel. It is found that strong and tough materials with primarily elastic response, like toughened ceramics, were required in order to enable dental crown/bridges to have long-term accuracy $[10,11]$. Also, the recently introduced nano-filled composite materials can be used in order to bond orthodontic brackets to teeth if its consistency could be more influx to readily adhere to the bracket base $[10$, $11,42]$.

Another example, effects of dental bleaching was tested on micro- and nanomorphological alterations of the enamel surface and found that that the thickness of the enamel smear layer was significantly reduced due to the bleaching process when nanomaterials used $[10,11]$.

According to the literature, there was study on the relative wear resistance of many kinds of denture teeth using an in vitro wear testing device and illustrated that, the nano-composite tooth was harder and more wearresistant in comparison with the acrylic teeth [10].

Nano-dentistry will make possible the maintenance of comprehensive oral health througth involving the use of nanomaterials, biotechnology (inclusive tissue engineering), and dental nanorobotics. It is another significant branch of medicine nowadays [42].

\subsubsection{Nanobiotechnology and gene therapy}

Gene therapy is the progressive therapeutic concept at present. Gene therapy describes local or systemic administration of a nucleic acid construct which can treat or prevent the diseases. Also, it includes cure diseases via changing the expression of genes that are responsible for the pathological case. This therapy is the hope for treatment of currently chronic diseases $[10,24,37]$. 
Nanobiotechnology can be functional for the complex gene therapy process. Presently, both biodegradable and non-biodegradable inorganic particles can be completely fabricated on the nano-scale with the attributes of binding DNA, internalizing across the plasma membrane and finally releasing in the cytoplasm for a protein final expression. In addition to the classical system (intravenous injection), development of polymer-based nanoparticle (NP) technologies for oral gene therapy is in continuous development [10,37]. As well as, Hatakeyama et al, are reported on development of a new systemic gene delivery system for cancer therapy with a tumourspecific cleavable PEG-lipid [11,37].

\subsection{Properties and Applications of nanoparticles in therapeutics, health and medicine}

Nanomaterials are investigated as promising tools for the development of diagnostic biosensors, and drug/gene delivery for their novel physicochemical and biological properties. Several properties of nanomaterials, including shape, size, chemical composition, surface structure, surface charge, agglomeration, aggregation, and solubility can greatly affect their interactions with biomolecules and cells [10].

Furthermore, the development of safe and simple methods to detect and treatment serious diseases, (such as cancer), is one of the biggest challenges for researchers in the biotechnology and medical research field nowadays [43]. Metal nanoparticles, such as $\mathrm{Au}$ (gold), $\mathrm{Ag}$ (silver), $\mathrm{Cu}$ (copper) and $\mathrm{Zn}$ (zinc) nanoparticles (NPs), have been broadly investigated over the past two decades due to their unique chemical and optical properties as mentioned early [44]. Nanotechnology has been applied in therapeutic treatment mostly in drug delivery and in cancer therapy [45]. This is because functionalised NPs can recognise and integrate with a biological matrix via the protecting ligands on NPs surface $[46,47]$. For many years, NPs have been tested in the therapeutic field to reduce side effects of cancer drugs via reducing dosage of cancer drugs. Some of the metal NPs have similar sizes when compared to some biomolecules. For instance, proteins have sizes $(1-20 \mathrm{~nm})$, cell surface receptors $(\sim 10 \mathrm{~nm})$, viruses $(\sim 20 \mathrm{~nm})$, DNA ( diameter $2 \mathrm{~nm})$, cell membrane $(\sim 6-10 \mathrm{~nm})$, and haemoglobin $(\sim 5 \mathrm{~nm})$ [4850]. As well as, NPs $(50 \mathrm{~nm})$ or less in size have shown capability to interact with DNA, enzymes, proteins, and cell receptors extracellularly and intracellularly in human cells, which are considered to be hundred to thousand times bigger than these NPs [48] (see Figure 2). For instance, NPs of $\leq 20 \mathrm{~nm}$ in diameter have discovered to exit blood vessels and circulate throughout the body [51]. During the last decade, AuNPs were seriously tested for biomedical applications, (such as gene, imaging, and photothermal therapies, and drug delivery) as a result to their novel chemical and optical properties, as well as their stability [52-55].

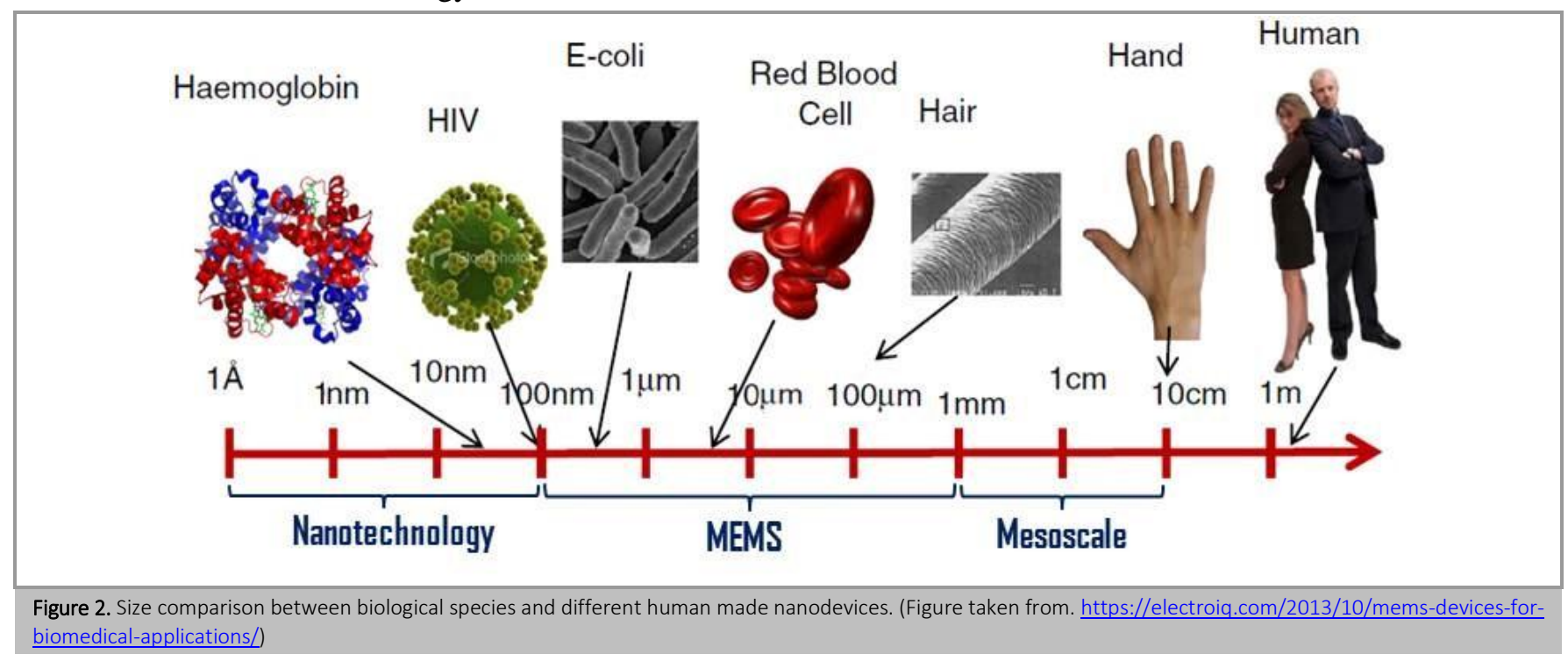

\subsection{Toxicology and safety of Nanostructures and Nanoparticles}

The effects of nanomaterials to on humans or the environment is still poorly understood but data on their possible effects is wanted so that expanded development and application of nanotechnology can proceed. For example, Magic Nano was rapid pulled as a nanotechnology-based product, which used in a spray-on ceramic sealant to repel dirt. As well as, over 110 consumers in Europe reported respiratory symptoms after the product was used and the product was pulled in March 2006 [56]. There is important needed to educate the public about these new 
technologies and to discuss their toxicity as well a potential safety issues and how these are being treated. Risk assessment of using nanomaterials attends some unique challenges due to there is little published researches on which to base conclusions and suggestions [57]. It is well known that, qualified thirteen experts in a different of relevant fields were interviewed to organise a list of factors affecting the potential human health risks and environmental risks of nanoparticles. Information was sorted into an impact diagram with relationships that the experts hypothesised would affect safety assessment. This framework can be useful and helping prioritise experiments necessary to define safety of nanoparticles. [58]. Modern advances in nanotechnology are helping evolution of sensors that define markers of exposure, biological responses, and environmental remediation [59]. Some consumer products (cosmetics, sunscreen, sports equipment, textiles) containing nanomaterials have been examined to assess the potential human exposure of nanoparticles of these products and any potential risks they pose [60]. In the other hand, different nanomaterial liposomes, cubosomes, solid lipid NP, dendrimers etc have advance properties for skin care. For example, nano emulsion is the most significant part in this regard and they are broadly used in lotion, gel, and creams emulsions. Sunscreen with inorganic material like metal $\mathrm{TiO}_{2}$ and $\mathrm{ZnO}_{2}$ found to be better in skin care products as mentioned in the literature [61].

\subsection{Toxicology}

It is well to mention that the other side to remove nanoparticles before delivery of the drug is the harmful toxicity associated with the nanoparticles themselves. Nanoparticles as a whole, or the decomposition products of these nanoparticles, are known to cause symptoms such as dermal changes, neurotoxicity, sensitization, growth toxicity, cardiac toxicity [62]. It should also be noted that studies that monitor the longterm effects of these nanoparticles are still ongoing and data are still collected on the toxic effects of some nanoparticles. Biodegradable Nanopolymers, such as polylactide (PLA), polyglycolic acid (PGA), and poly(lactic-co-glycolic acid) (PLGA) can be fully dissolved and widely used for the packaging of many anticancer drugs to minimize toxicity [62].

Nanoparticles in the bloodstream are alien organisms and undergo different ways of getting rid of the body, such as the immune system, organs such as kidneys and liver $[10,11]$. In order to effectively uptake the tumor, nanoparticles need to overcome these cleansing forces that are actively driving them out of the body. The single-core phagocytic system contains of phagocytic cells, such as macrophages, Kupffer cells, and monocytes found in organs like the liver, spleen and bone marrow that can engulf nanoparticles and make degradation $[11,63]$.

The phagocytes recognize nanoparticles through the presence of opsonin such as immunoglobulin proteins, complement proteins, albumins, fibronectin, and apolipoproteins found in the serum. Once nanoparticles are opsonized and engulfed, they are subjected to enzymatic degradation, with non-degradable parts being sent to organs such as the liver and spleen. Kupffer cells and hepatocytes cells in the liver are used in the cleaning of NPs products [60]. NPs that are larger than $250 \mathrm{~nm}$ accumulate and are cleared by the spleen [62]. Various ways were used to reduce the clearance of NPs before delivering their drug cargo. The charged NPs are opsonized faster than the neutral particles [11, $63,64]$. Therefore, by neutralizing the surface charge, shading can be minimized. In addition, coating nanoparticles with PEG reduces opsonization, which causes increasing the plasma rotation time of nanoparticles. Kidney removal is a volume-based filtration process where the kidneys remove nanoparticles smaller than 8 nanometer [63].

\section{Conclusions}

Many areas of science converge to study science at a basic or block level, namely nanoscience. Most studies focused on materials science with some emerging applications in the field of biomedicine. Some of basic studies have been conducted in the field of medicines to discuss the fundamentally different properties of nanoparticles. The applications of nonmaterial compounds are clear and promising. However, the toxicity of nanomaterials is still poorly understood.

The review of available literature has revealed a great deal of literature on this subject, and the literature describes the gradual evolution of the concept of nanotechnology and its applications.

Based on a review of available literature, the following concluding observations are made: Developers and researchers of nanotechnology concepts should work together with more application-based researchers to ensure that developments can lead to useful products and the nano science seems poised to be leading to a revolution in the world of medicine.

\section{Acknowledgment}

The author is thankful to the Sebha University, Chemistry Department for its support.

\section{References}

[1] Jennings, C. H. (2009). Nanotechnology in the USA: Developments, Policies and Issues. Nova Science Publishers, Incorporated.

[2] Trgovcević, Z. (1994). 50 years of molecular biology. Lijecnicki vjesnik, 116(11-12), 315-318. 
[3] Lewin R. (1987). National Academy looks at human genome. Science, Feb13; 235 (4790). 7478.

[4] Johnson, R. S. (1987). The human genome project: what impact on basic research?. The FASEB Journal, 1(6), 502-505.

[5] Yang, Z. R., \& Hamer, R. (2007). Bio-basis function neural networks in protein data mining. Current pharmaceutical design, 13(14), 14031413.

[6] Ahrens, C. H., Wagner, U., Rehrauer, H. K., Türker, C., \& Schlapbach, R. (2007). Current challenges and approaches for the synergistic use of systems biology data in the scientific community. In Plant Systems Biology. Birkhäuser Basel, 277-307.

[7] ordan, B. R. (1999). 'Genomics': Buzzword or reality? Journal of biomedical science, 6(3), 145150.

[8] Weyant, R. G. (1967). Lycurgus: The father of applied psychology? American Psychologist, 22(6), 432.

[9] Tolochko, N. K. (2009). History of nanotechnology. Encyclopedia of Life Support Systems (EOLSS).

[10] Logothetidis, S. (Ed.). (2012). Nanomedicine and nanobiotechnology. Springer Science \& Business Media.

[11] Bawa, R., Audette, G. F., \& Rubinstein, I. (2016). Handbook of clinical nanomedicine: nanoparticles, imaging, therapy, and clinical applications. Pan Stanford.

[12] Hulla, J. E., Sahu, S. C., \& Hayes, A. W. (2015). Nanotechnology: History and future. Human \& experimental toxicology, 34(12), 13181321.

[13] Jain, K. K. (2004). Applications of biochips: from diagnostics to personalized medicine. Current opinion in drug discovery \& development, 7(3), 285-289.

[14] Brown, E. M. B. (2014). Nanomedicine Advancements in Cancer Diagnosis and Treatment. Horizons in Clinical Nanomedicine, 67.

[15] Emerich DF. (2005). Nanomedicineprospective therapeutic and diagnostic applied usages. Expert Opin. Biol. Ther, Jan;5(1). 1-5.

[16] Avti, P. K., Patel, S. C., \& Sitharaman, B. (2011). Nanobiomaterials: current status and future prospects. Nanobiomaterials handbook Edited by Balaji Sitharaman. CRC publication.

[17] Lee, L. A., \& Wang, Q. (2006). Adaptations of nanoscale viruses and other protein cages for medical applications. Nanomedicine: Nanotechnology, Biology and Medicine, 2(3), 137149.
[18] Murthy, S. K. (2007). Nanoparticles in modern medicine: state of the art and future challenges. International journal of nanomedicine, 2(2), 12941.

[19] Pauwels, E. K., \& Erba, P. (2007). Towards the use of nanoparticles in cancer therapy and imaging. Drug news \& perspectives, 20(4), 213-220.

[20] Moghimi, S. M., Hunter, A. C., \& Murray, J. C. (2005). Nanomedicine: current status and future prospects. The FASEB journal, 19(3), 311-330.

[21] Gulati, N., \& Gupta, H. (2012). Nanomedicine: Potential devices for diagnostics. Recent Patents on Nanomedicine, 2(2), 146-155.

[22] Nakayama, M., \& Okano, T. (2005). Drug delivery systems using nano-sized drug carriers. Gan to kagaku ryoho. Cancer \& chemotherapy, 32(7), 935-940.

[23] Dutta, R. C. (2007). Drug carriers in pharmaceutical design: promises and progress. Current pharmaceutical design, 13(7), 761-769.

[24] Dobson, J. (2006). Magnetic micro-and nanoparticle-based targeting for drug and gene delivery. Nanomedicine.

[25] Freitas, R. A. (2005). Nanotechnology, nanomedicine and nanosurgery. International Journal of Surgery, 4(3), 243-246.

[26] Ebbesen, M., \& Jensen, T. G. (2006). Nanomedicine: techniques, potentials, and ethical implications. BioMed Research International, 2006.

[27] Fankhauser, F., Niederer, P. F., Kwasniewska, S., \& van der Zypen, E. (2004). Supernormal vision, high-resolution retinal imaging, multiphoton imaging and nanosurgery of the cornea-a review. Technology and Health Care, 12(6), 443-453.

[28] Lamounier, J. A., Moulin, Z. S., \& Xavier, C. C. (2004). Recommendations for breastfeeding during maternal infections. Jornal de pediatria, 80(5), s181-s188.

[29] Iwaki, Y., Aiba, N., Tran, H. T. T., Ding, X., Hayashi, S., Arakawa, Y., \& Abe, K. (2003). Simian TT virus (s-TTV) infection in patients with liver diseases. Hepatology research, 25(2), 135142.

[30] Pawłowska, M., \& Halota, W. (2004). HCV infections in view of clinical practice. Medical Science Monitor, 10(1), 24-28.

[31] Filteau, S. M. (2000). Role of breast-feeding in managing malnutrition and infectious disease. Proceedings of the Nutrition Society, 59(4), 565572.

[32] Lawrence, R. M., \& Lawrence, R. A. (2004). Breast milk and infection. Clinics in perinatology, 31(3), 501-528. 
[33] Rajabi, M., Signorelli, P., Gorincioi, E., Ghidoni, R., \& Santaniello, E. (2010). Antiproliferative activity of N 6isopentenyladenosine on MCF-7 breast cancer cells: cell cycle analysis and DNA-binding study. DNA and cell biology, 29(11), 687-691.

[34] Sahoo, S. K., \& Labhasetwar, V. (2003). Nanotech approaches to drug delivery and imaging. Drug discovery today, 8(24), 1112-1120.

[35] Smith, D. J. (2007). Characterization of nanomaterials using transmission electron microscopy. The Royal Society of Chemistry: Cambridge.1-27.

[36] Angeli, E., Buzio, R., Firpo, G., Magrassi, R., Mussi, V., Repetto, L., \& Valbusa, U. (2008). Nanotechnology applications in medicine. Tumori Journal, 94(2), 206-215.

[37] Rajabi, M., Srinivasan, M., \& Mousa, S. A. (2016). Nanobiomaterials in drug delivery. In Nanobiomaterials in Drug Delivery. William Andrew Publishing. 1-37.

[38] Allen, T. M., \& Martin, F. J. (2004, December). Advantages of liposomal delivery systems for anthracyclines. In Seminars in oncology. WB Saunders. (31), 5-15.

[39] Bhattacharyya, P., Pradhan, K., Paul, S., \& Das, A. R. (2012). Nano crystalline $\mathrm{ZnO}$ catalyzed one pot multicomponent reaction for an easy access of fully decorated 4H-pyran scaffolds and its rearrangement to 2-pyridone nucleus in aqueous media. Tetrahedron Letters, 53(35), 4687-4691.

[40] Heravi, M. M., Oskooie, H. A., Karimi, N., \& Hamidi, H. (2011). KAl (SO4) 2•12H2O catalyzed efficient synthesis of 3, 4, 6-trisubstituted 2pyridone in water. Chinese Chemical Letters, 22(9), 1059-1062.

[41] Louis, H., Fidelis, G. K., Fidelis, T. T., \& Onoshe, S. (2019). Curcumin and Curcumin-based derivatives as anti-cancer agents: Recent NanoSynthetic Methodologies and Anti-cancer Therapeutic Mechanisms. Journal of Medicinal and Chemical Sciences, 2(41-75.), 59-63.

[42] Freitas Jr, R. A. (2000). Nanodentistry. The Journal of the American Dental Association, 131(11), 1559-1565.

[43] Yao, J., Yang, M., \& Duan, Y. (2014). Chemistry, biology, and medicine of fluorescent nanomaterials and related systems: new insights into biosensing, bioimaging, genomics, diagnostics, and therapy. Chemical reviews, 114(12), 6130-6178.

[44] Shan, J., \& Tenhu, H. (2007). Recent advances in polymer protected gold nanoparticles: synthesis, properties and applications. Chemical Communications, (44), 4580-4598.
[45] Cai, W., Gao, T., Hong, H., \& Sun, J. (2008). Applications of gold nanoparticles in cancer nanotechnology. Nanotechnology, science and applications, 1, 17.

[46] De Jong, W. H., \& Borm, P. J. (2008). Drug delivery and nanoparticles: applications and hazards. International journal of nanomedicine, 3(2), 133.

[47] Sapsford, K. E., Algar, W. R., Berti, L., Gemmill, K. B., Casey, B. J., Oh, E., \& Medintz, I. L. (2013). Functionalizing nanoparticles with biological molecules: developing chemistries that facilitate nanotechnology. Chemical reviews, 113(3), 1904-2074.

[48] Gao, J., Huang, X., Liu, H., Zan, F., \& Ren, J. (2012). Colloidal stability of gold nanoparticles modified with thiol compounds: bioconjugation and application in cancer cell imaging. Langmuir, 28(9), 4464-4471.

[49] Alexis, F., Rhee, J. W., Richie, J. P., RadovicMoreno, A. F., Langer, R., \& Farokhzad, O. C. (2008, January). New frontiers in nanotechnology for cancer treatment. In Urologic Oncology: Seminars and Original Investigations. Elsevier. 26(1), 74-85.

[50] Loos, M. (2014). Carbon nanotube reinforced composites: CNT Polymer Science and Technology. Elsevier.

[51] Ju-Nam, Y and Lead, J. (2016). Properties, Sources, Pathways, and Fate of Nanoparticles in the Environment. Engineered Nanoparticles and the Environment: Biophysicochemical Processes and Toxicity. 39-117.

[52] Patra, C. R., Bhattacharya, R., Mukhopadhyay, D., \& Mukherjee, P. (2010). Fabrication of gold nanoparticles for targeted therapy in pancreatic cancer. Advanced drug delivery reviews, 62(3), 346-361.

[53] Zhao, Y., Gu, X., Ma, H., He, X., Liu, M., \& Ding, Y. (2011). Association of glutathione level and cytotoxicity of gold nanoparticles in lung cancer cells. The Journal of Physical Chemistry C, 115(26), 12797-12802.

[54] Medley, C. D., Smith, J. E., Tang, Z., Wu, Y., Bamrungsap, S., \& Tan, W. (2008). Gold nanoparticle-based colorimetric assay for the direct detection of cancerous cells. Analytical chemistry, 80(4), 1067-1072.

[55] Cheng, Y., Samia, A. C., Meyers, J. D., Panagopoulos, I., Fei, B., \& Burda, C. (2008). Highly efficient drug delivery with gold nanoparticle vectors for in vivo photodynamic therapy of cancer. Journal of the American Chemical Society, 130(32), 10643-10647.

[56] Piller, C. (2006). Science's tiny, big unknown. Los Angeles Times, 1, A1. 
[57] Morgan, K. (2005). Development of a preliminary framework for informing the risk analysis and risk management of nanoparticles. Risk Analysis: An International Journal, 25(6), 1621-1635.

[58] Borm, P., Klaessig, F. C., Landry, T. D., Moudgil, B., Pauluhn, J., Thomas, K., \& Wood, S. (2006). Research strategies for safety evaluation of nanomaterials, part $\mathrm{V}$ : role of dissolution in biological fate and effects of nanoscale particles. Toxicological Sciences, 90(1), 23-32.

[59] Balshaw, D. M., Philbert, M., \& Suk, W. A. (2005). Research strategies for safety evaluation of nanomaterials, Part III: nanoscale technologies for assessing risk and improving public health. Toxicological Sciences, 88(2), 298-306.

[60] Thomas, T., Thomas, K., Sadrieh, N., Savage, N., Adair, P., \& Bronaugh, R. (2006). Research strategies for safety evaluation of nanomaterials, part VII: evaluating consumer exposure to nanoscale materials. Toxicological Sciences, 91(1), 14-19.
[61] Hameed, A., Fatima, G. R., Malik, K., Muqadas, A., \& Fazal-ur-Rehman, M. (2018). Scope of Nanotechnology in Cosmetics: Dermatology and Skin Care Products. Journal of Medicinal and Chemical Sciences, 2(1), 9-16.

[62] Love, S. A., Maurer-Jones, M. A., Thompson, J. W., Lin, Y. S., \& Haynes, C. L. (2012). Assessing nanoparticle toxicity. Annual review of analytical chemistry, 5, 181-205.

[63] Lee, S. Y., \& Cheng, J. X. (2010). Clearance of nanoparticles during circulation. Pharmaceutical Sciences Encyclopedia: Drug Discovery, Development, and Manufacturing, 1-32.

[64] Johnston, H. J., Semmler-Behnke, M., Brown, D. M., Kreyling, W., Tran, L., \& Stone, V. (2010). Evaluating the uptake and intracellular fate of polystyrene nanoparticles by primary and hepatocyte cell lines in vitro. Toxicology and applied pharmacology, 242(1), 66-78.

How to cite this manuscript: Wanisa Abdussalam-Mohammed, Review of Therapeutic Applications of Nanotechnology in Medicine Field and its Side Effects, Journal of Chemical Reviews, 2019, 1(3), 243-251. 